\title{
Overview of Rubik's Cube and Reflections on Its Application in Mechanism
}

\author{
Da-Xing Zeng, Ming Li, Juan-Juan Wang, Yu-Lei Hou, Wen-Juan Lu and Zhen Huang*
}

\begin{abstract}
Rubik's Cube is a widely popular mechanical puzzle that has attracted attention around the world because of its unique characteristics. As a classic brain-training toy well known to the public, Rubik's Cube was used for scientific research and technology development by many scholars. This paper provides a basic understanding of the Rubik's Cube and shows its mechanical art from the aspects of origin and development, characteristics, research status and especially its mechanical engineering design, as well as making a vision for the application in mechanism. First, the invention and origin of Rubik's Cube are presented, and then the special characteristics of the cube itself are analyzed. After that, the present researches of Rubik's Cube are reviewed in various disciplines at home and abroad, including the researches of Rubik's Cube scientific metaphors, reduction algorithms, characteristic applications, and mechanism issues. Finally, the applications and prospects of Rubik's Cube in the field of mechanism are discussed.
\end{abstract}

Keywords: Rubik's Cube, Scientific research, Mechanical art, Application and prospects

\section{Introduction}

Rubik's Cube is a 3D combination puzzle invented in 1974 by Hungarian sculptor and professor of architecture Ernő Rubik [1] and was originally called the Magic Cube $[2,3]$. This invention caused the widespread interest in the world owing to its unique characteristics, which exerted a profound impact on mankind. Rubik's Cube is listed as one of the 100 most influential inventions during the 20th century [4]. Additionally, it is widely considered to be the world's best-selling toy [5]. It won a German Game of the Year special award [6] and won similar awards for best toy in the UK, France, and the US. [7].

Although the Rubik's Cube reached its height of mainstream popularity in the 1980 s, it is still widely known and used. It not only attracts Rubik's Cube enthusiasts conducting research into Rubik's Cube reduction algorithms [8-10] but also draws the attention of scientists and technical workers from various walks of life for its sophisticated design and ideas [11]. On the one hand, the Rubik's Cube structure has several features such as rotation, permutation and combinations, and cycle and

*Correspondence: huangz@ysu.edu.cn

School of Mechanical Engineering, Yanshan University, Qinhuangdao 066004, China symmetry, which were treated as physical models or tools to study specific scientific issues or were studied by using scientific theory or methods in some areas. All in all, the principles of Rubik's Cube are contained in numerous scientific systems that involve permutations and combinations, symmetries, and cyclicality. On the other hand, scholars began to explore the inner movement principles of the Rubik's Cube structure. The application prospects of Rubik's Cube have been discussed according to its rotation characteristics.

\section{Origin and Development of Rubik's Cube}

The concept of Rubik's Cube originated from the China Luo Book [12], which can be simplified into a Jiugong map, as shown in Figure 1.

Jiugong map is a zero-dimensional third-order cube. The $n$-order magic square is a certain configuration formed by a combination of numbers $1,2,3, \ldots, n^{2}$ in an $n$-order square, which makes the sum of the numbers in each row, column, and two diagonal lines $n\left(n^{2}+1\right) / 2$. This is called a magic square constant. The magic square constant of a third-order cube is 15 [13].

Rearrange Jiugong is a one-dimensional third-order cube and is a game developed from the Jiugong map approximately during the Chinese Yuan Dynasty. This 


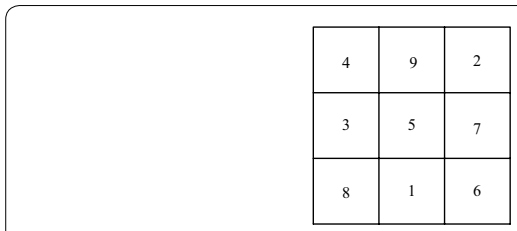

Figure 1 Jiugong map

game places eight movable pieces in the nine places in the board by moving the pieces to create another pattern in order to complete the pattern change of Rearrange Jiugong.

Rearrange Jiugong spread to the west, and Sam Loyd invented the 15 chess on its basis. The principle of 15 chess (to move the pieces to achieve pattern changes) is the same as that of Rearrange Jiugong. However, the order of 15 chess is a four-order cube that is one order higher than Rearrange Jiugong.

After that, the development of the cube shifted from the order to the dimension. In the Qing Dynasty, Chinese scholars put forward the thought of using digital pieces of three-dimensional magic squares. In fact, this was a three-dimensional second-order cube prototype, as shown in Figure 2.

The three-dimensional magic square did not have a rotational characteristic until Rubik designed a rotatable mechanics cube in 1974. Japanese Stonefur completed the same design alone in 1976 . The principle of moving the pieces to achieve pattern changes is similar to Rearrange Jiugong, through moving pieces to achieve overall changes. The core principle is that the movement path of a piece is constrained by other pieces that are in contact with the one.

Rubik made the first three-order cube in the world, which looks like a sphere and is operated by constraints between the components to achieve a particular rotation. This is shown in Figure 3. Initially, taking security issues into account, the eight vertices of the cube were cut a bit. Later, it was changed into sharp corners and became a cubic shape because its vertex did not affect the appearance and the production process became simpler. Today's common third-order cube is shown in

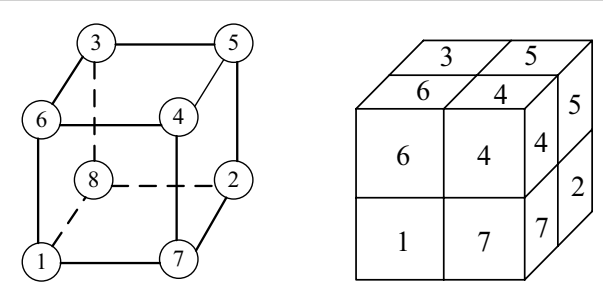

Figure 2 Three-dimensional magic square

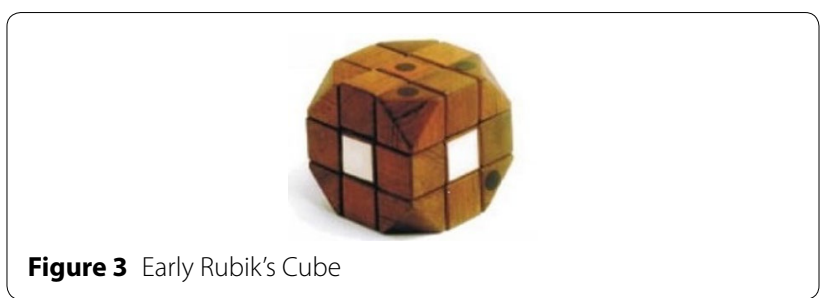

Figure 4. It can be seen that the shape of Rubik's Cube can be redesigned without affecting the rotation of the cube structure.

A variety of modified cubes have been designed to expand the cube family after Rubik's Cube was invented [14]. Generally, the magic cube can be divided into two categories: cubic cube and specially shaped cube. A cubic cube refers to a cube in a box structure that is not changed, but the cube's order increases. There are different variations of Rubik's Cubes with up to 33 layers, with $2 \times 2 \times 2$ (Pocket/Mini Cube), standard $3 \times 3 \times 3$ cube, $4 \times 4 \times 4$ (Rubik's Revenge/Master Cube), and $5 \times 5 \times 5$ (Professor's Cube) the most well known. The $17 \times 17 \times 17$ "Over the Top" cube (available late 2011) was until December 2017 the largest (and most expensive, costing more than US $\$ 2,000$ ) commercially sold cube. A working design for a $22 \times 22 \times 22$ cube exists and was demonstrated in January 2016 [15], and a $33 \times 33 \times 33$ cube was introduced in December 2017 [16]. Chinese manufacturer ShengShou has been producing cubes in all sizes from $2 \times 2 \times 2$ to $10 \times 10 \times 10$ (as of late 2013) [17], and also produced an $11 \times 11 \times 11$ model. Figure 5 lists six cases of the cube.

The specially shaped cube refers to the cube family besides the cubic cube. The structural shape of specially shaped cube is diverse, including polyhedron cubes, sphere cube, tetrahedron cube, mirror cube, gear cube, cake cube, and so on. Figure 6 lists six cases of specially shaped cubes.

As early as 1959, a second-order cube whose rotation relies on magnetism was invented. However, this cube is less popular than Rubik's Cube, whose rotation relies on mechanical in the later. On the one hand, the cube's cost is too high owing to its magnetic structure and prevailing market economy factors. On the other hand, the second-order cube is difficult to restore. Next, Rubik invented the third-order cube, which has a compact structure, clever design, and low cost, thus overcoming the congenital deficiencies of the second-order magnetic cube. In short, the special mechanical structure inside is the key to the Rubik's Cube's ingenuity. 


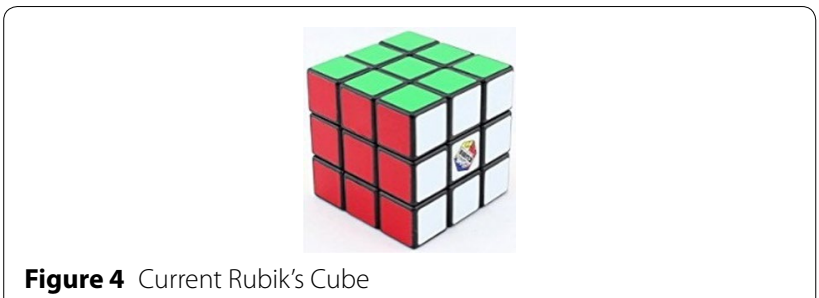

Figure 4 Current Rubik's Cube
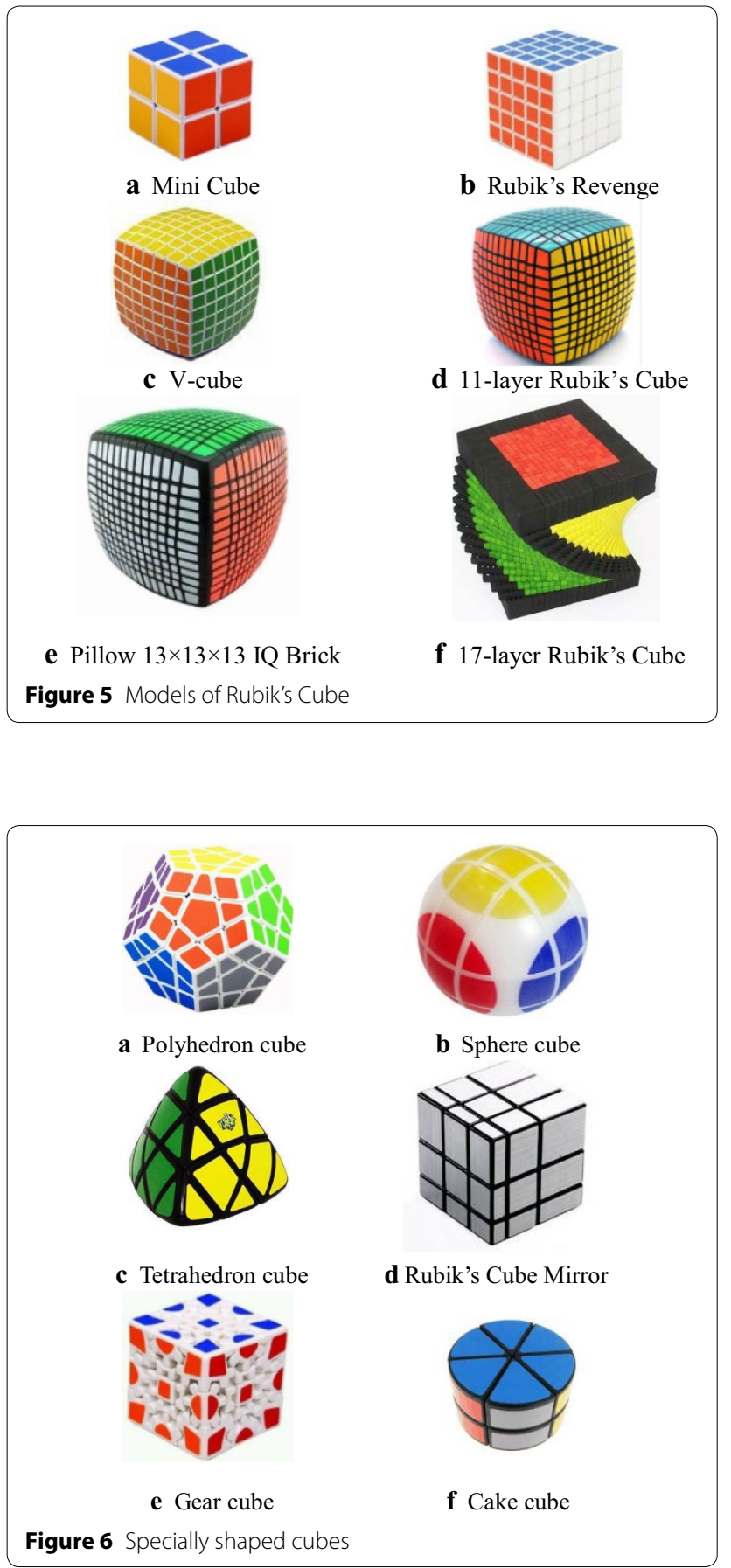

\section{Characteristics of Rubik's Cube}

\subsection{Structural Rotational Characteristic}

The structure of the Rubik's Cube mechanism is the most wonderful aspect of the Cube. At first, it seems completely impossible for the Cube to work. Very few people proposed a possible mechanism, and very few of these were practical. Later, many mechanisms of Rubik's Cube were based on mortise and tenon connections between pieces, as shown in Figure 7.

Rubik's mechanism has connections as shown in Figure 7 (c), which greatly simplify the shapes of the pieces. The mortise and tenon methods can be used so that the 26 exterior pieces of the Cube are held together with no central piece at all. This connection is difficult to make with sufficient accuracy so that the faces will turn easily [18].

In Figure 8, the standard $3 \times 3 \times 3$ cube consists of 26 unique miniature cubes, also called "pieces" or "cubelets" including a center shaft frame, 6 center pieces, 8 corner pieces, and 12 edge pieces. The various types of pieces of Rubik's Cube are shown in Figure 9. Each of these includes a concealed inward extension that interlocks with the other pieces while permitting them to move to different locations.

Figure 10 shows that the center piece can be split into two parts. The center piece and the center shaft frame are connected by spring-loaded screws, which are shown in Figure 11.

Figure 12 introduces the process of assembling different pieces one by one. The constraints on the edges are formed by structural restrictions and the force locking of two adjacent centers. The constraints on the corner piece are formed by structural restrictions and the force locking of three adjacent edges.

The contact modes between the centers and edges, and the edges and corners, are shown in Figure 13. All of them contact each other by a plane and a curved surface.

The feet (inward extension) of corner pieces and edge pieces are tightly hooked together. These form a chuck that is similar to the clamping part of a machine, as shown in Figure 14(a). On the one hand, nine small pieces as shown in Figure 14(b) are tightly clamped so that they cannot spread out. On the other hand, the Rubik's Cube layer can rotate around the shaft flexibly when force is applied.

The characteristic of Rubik's Cube wherein small pieces are joined together by a mutual mosaic is similar to the Luban lock, which was invented based on the principle of gossip metaphysics. The Luban lock is a traditional Chinese intelligent toy. It originated from the skeletal structures of Chinese ancient architecture. Its parts are held together by structural restriction without any nails 

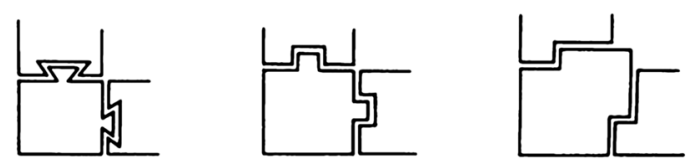

a Dovetail tenon b Square tenon

c Shouldered tenon

Figure 7 Three kinds of mortise and tenon connections of Rubik's Cube

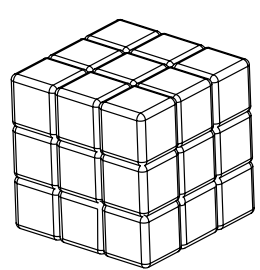

Figure 8 Rubik's Cube

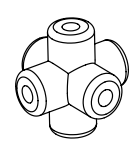

a Center shaft frame

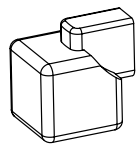

c Edge piece

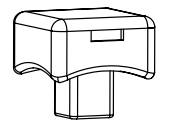

b Center piece

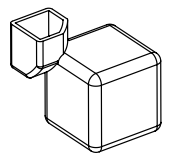

d Corner piece
Figure 9 Unit structure of Rubik's Cube

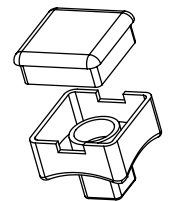

Figure 10 Disassembled center piece

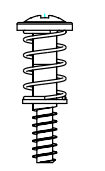

Figure 11 Spring-loaded screws or ropes. The relationship between different parts is complicated.

Figure 15 introduces a ball Luban lock, which is assembled by six wood can be fitted together. The six wooden parts are shown in Figure 16. In the assembly, the wooden parts are fastened together, leaving space for the insertion of the rear components until the last part of the "keyhole" is placed. The entire Luban lock is firmly held together, with the parts restraining each other and supporting each other. The combined structure of the Rubik's Cube is derived from the squatting structure of the Luban lock.

Six cases of Luban lock structure are shown in Figure 17. The mortise and tenon structures are connected by the joining method of concavo-convex treatment. This shows the great creativity and achievements of ancients.

The stability of the mortise and tenon structure is affected by factors including the place of grooves, the density of the patchwork, and the degree of trenching tightness. The success of connecting a mortise and tenon structure depends on how differently sized pieces are placed together precisely. The entire lock will fall to pieces when a wooden bar is removed. Both the Luban lock and Rubik's Cube use mosaic structures to achieve overall stability. The difference between Rubik's Cube and the Luban lock is that Rubik's Cube can turn a layer by exerting force on it, while the Luban lock cannot.

\subsection{Permutation and Combination Characteristic}

Combinatorics originated from the Eastern Luo book, and the idea of permutation and combination was proposed to meet the need of human counts [13]. The ancient Chinese eight diagrams, calendar of Heavenly stems and Earthly branches, and some divination methods all contain the concepts of permutation and combination. Eight diagrams can describe the space coordinate system of Rubik's Cube, and correspond to the Cartesian coordinate system one to one. These show that the rotation transformation of Rubik's Cube contains the ideas of permutation and combination. Rubik's Cube achieves the desired state and all kinds of combinations of colors by rotating pieces.

The original $(3 \times 3 \times 3)$ Rubik's Cube has six faces. Initially, each face has the same color, and each face has nine small outer surfaces. They are 54 outer surfaces in total. Every side of Rubik's Cube is composed of diversiform color pieces after the random rotation of different sides several times.

The original $(3 \times 3 \times 3)$ Rubik's Cube has 8 corners and 12 edges. Corners can be arranged in 8 ! $(40,320)$ ways, and there are $3^{7}$ (2187) possible orientations because the orientation of the eighth (final) corner depends on the preceding ones. Edges can be arranged by $12 ! / 2$ $(239,500,800)$ ways, restricted from 12 ! because edges 

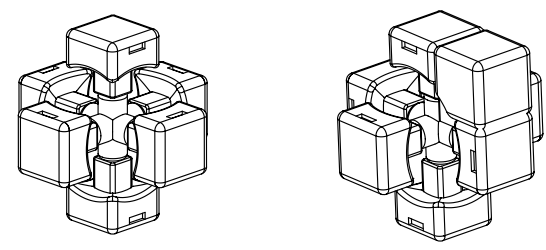

a Connection between six centers and center shaft frame

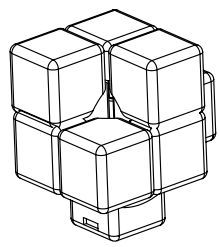

c Connection between three centers and three edges b Connection between two centers and one edge

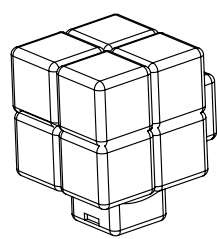

d Connection between three edges and one corner

Figure 12 Assembly process of four types of pieces

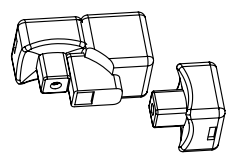

a Contact mode between centers and edges

Figure 13 Contact mode between different pieces

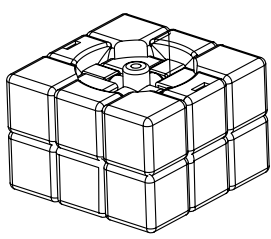

a Inner chuck form of Rubik's Cube

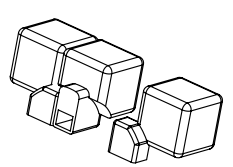

b Contact mode between edges and corners
Figure 14 Inner structure of Rubik's Cube

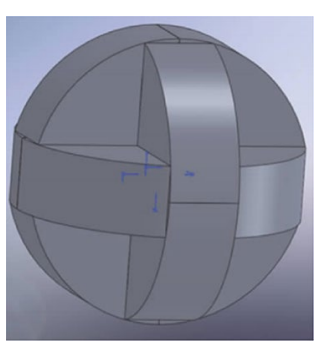

Figure 15 Ball Luban lock after assembly must be in an even permutation exactly when the corners are. There are $2^{11}$ (2048) possibilities because 11 edges can be flipped independently, with the flip of the 12th depending on the preceding ones. The number of Rubik's Cube configuration species is about

$$
8 ! \times 3^{7} \times(12 ! / 2) \times 2^{11}=43252003274489856000
$$

which is approximately 43 quintillion [19].

The transformation among different configurations of Rubik's Cube can be used as a mode that uses the idea of permutation and combinations to pursue the diversity of answers. The characteristics of combination and transformation inspire product design thinking, and have practical application value especially in industrial product modularization and structural design [20].

\subsection{Cycle Characteristic}

Circulation is one of the basic characteristics of Rubik's Cube. If using an arbitrary operations sequence (rotation sequence) to operate the cube cyclically, it will achieve cycle status. The cycle characteristic of operation can be divided into two categories: periodic and nonperiodic [12].

Assuming that Rubik's Cube is operated by certain number operations and specified operations from the original state back to the original state again, this means the Rubik's Cube achieves a cycle. The circulation period is the number of times the Rubik's Cube is rotated. If the circulation period is a constant, the cycle is periodic. If the circulation period is variable, the cycle is nonperiodic. The sequence of operations of a nonperiodic cycle is based on both the sequence of periodic cyclic operations and the "constant process" sequence of operations that causes the cube's pattern to exhibit a nonperiodic change. The operation sequence of "constant process" refers to the fact that all pieces can return to their original state after this sequence of operations.

\section{Research Status of Rubik's Cube}

Following the successful invention of the first Rubik's Cube, a group of commercial cubes was made in Hungary. Rubik's Cube was rated as the best toy at the international fair in Nuremberg Germany in 1978, and its inventor Rubik was awarded the "World's Best Game Invention Award." Since then, the popularity of Rubik's Cube has spread around the world. At the same time, an international mathematician representative conference was held in Helsinki. Rubik's Cube attracted great attention with the experts and researchers. After that, articles and writings about Rubik's Cube have been published continuously, and the door to explore the mysteries of Rubik's Cube was opened. 


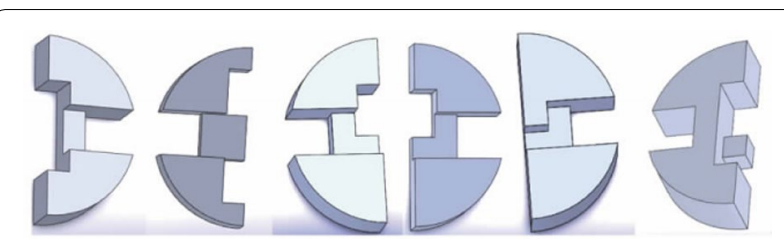

Figure 16 Ball Luban lock parts

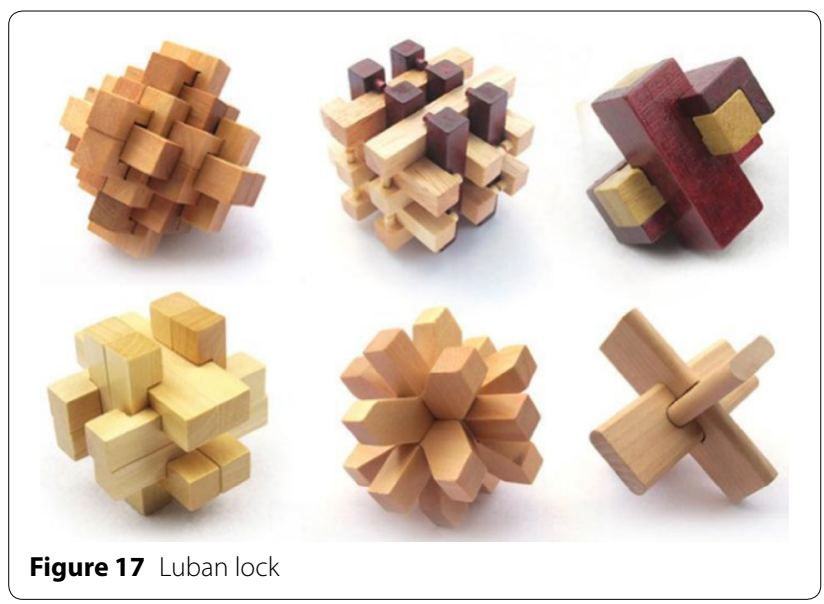

\subsection{Scientific Metaphor of Rubik's Cube}

The scientific metaphor of Rubik's Cube has two implications. One is that the problem of the cube is studied by using existing scientific theory or methods. David $[21,22]$ put forward a set of standard symbols of Rubik's Cube with regard to combinatorics and group theory. The rotation and cycle transposition of structure is the best interpretation of the concept and theorem of group theory. Li [12] used crystallographic symbols to establish a mathematical model that described an $n$-order cube based on the point group theory. A computer program of solving the cube problem was produced on the basis of the mathematical model.

Rubik's Cube is treated as an abstract tool to study specific scientific issues. In the literature [12], Rubik's Cube was used as a model to study crystal electron diffraction, chaos, genetic, and other scientific issues. American mathematician Joyner David [23] discussed group theory based completely on Rubik's Cube and other similar mathematical toys. Chen [24] studied Rubik's Cube rotation and defined the concept of the Rubik's Cube group.

According to the cube's rotation law, many properties of the Rubik's Cube group are described in the group theory system. Rubik's Cube is used as a model to describe the basic particles in physics. In addition, the structures of some substances in nature are similar to the Rubik's
Cube structure, including $\mathrm{Ag} / \mathrm{AgCl}[25]$ and the $\mathrm{TiO}_{2}$ nanocage [26], as shown in Figure 18.

In addition, Rubik's Cube also has some applications in the field of psychotherapy [27]. Rubik's Cube has gradually been taken seriously in the field of education because it contains rich scientific knowledge. Some primary and secondary schools carry out cube-based mathematics courses to improve students' learning ability. There are also some relevant studies showing that the use of "play cubes" as a teaching guide in primary school mathematics activities is conducive to stimulating students' interest in learning and guiding students to think abstractly and develop spatial ideas [28]. Rubik's Cube was viewed as a tool in applying rather sophisticated mathematics to generate some solution algorithms [29].

\subsection{Algorithm of Rubik's Cube}

Rubik's Cube has been known as a classic educational toy with a unique charm since it was invented. From general recovery to quick speed and least step to restore, from one hand twisted to blind recovery [30], more and more Rubik's Cube enthusiasts and racing players are keen on the higher challenges of playing Rubik's Cube.

There are many kinds of Rubik's Cube solutions. The "layer first law" was proposed by David Singmaster and is used by the most people. First, restore the top layer, and then restore the middle layer, and finally restore the bottom layer as well. Mathematician Conway [31] at Cambridge University proposed a six-stage reduction method from the bottom of the cube to the middle and then to the top, and from the edge pieces to the corner pieces gradually. Patrick [32] proposed a six-sided reduction method: first, restore the corner pieces and edge pieces of the top layer, then restore the bottom corner pieces, and finally the edge pieces of the middle and bottom are restored. Jessica invented the "Fridrich Method" which is

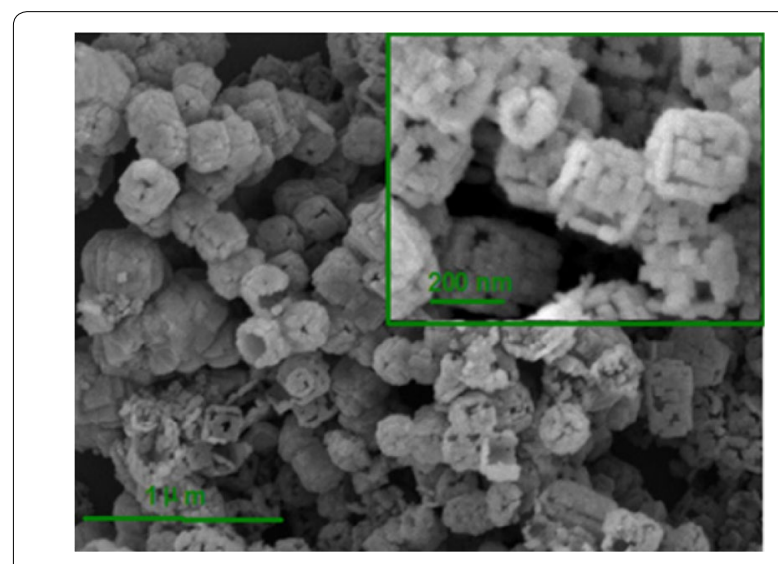

Figure $18 \mathrm{TiO}_{2}$ nanocages with Rubik-like structure 
used by most of the racing players to quickly restore the Rubik's Cube. This method is used widely, inspires and influences cube researchers, and has played a critical role in the development of Rubik's Cube [33].

The algorithm of Rubik's Cube can be solved by computer artificial intelligence as well. Richard E. Korf used the "macro operator" technique to solve the algorithm of Rubik's Cube by a computer program, and obtained a complete summary of the cube-side reduction process [34]. Rokicki et al. proved that the maximum number of moves to solve the cube is 20 [35].

\subsection{Application of Rubik's Cube Characteristics}

In recent years, with the development of broadband networks and the arrival of the information age, the security of data information and the protection of privacy rights are becoming more and more important. When Rubik's Cube stays in the initial state, all patches of each face are in the same color and will become chaotic after several rotations. The Rubik's Cube recovery can follow the reverse step of rotation or can follow the decomposition steps of restoring the Rubik's Cube. Thus, the rotated sequence can be regarded as an encrypted key to encrypt information or hide information. Otherwise, decryption is very difficult [36].

In Refs. [37-39], a chaotic sequence was used as the transform parameter of the Rubik's Cube, and the encryption method of the image was realized by a cyclic shift algorithm. In Ref. [40], an efficient method for image encryption based on the Rubik's cube principle with a chaotic Baker map was presented. The proposed technique enhanced the security level of the Rubik's cube encryption technique.

In addition, a chaotic mixing algorithm based on the concept of the Rubik's cube was proposed for watermarking images [41]. Ref. [42] used the matrix and threedimensional structure of a Rubik's Cube reference to achieve information hiding of grayscale images.

In Ref. [43], Tsai proposed multi-morphological image date hiding based on the application of a Rubik's Cube algorithm. An image can be partitioned into 54 units and form a Rubik's Cube. A total of 54 units is selected sequentially and transformed into 6 faces according to the 6 faces of a Rubik's Cube by designating an index number, as shown in Figure 19 and Figure 20. The encrypted data is scrambled by performing a Rubik's Cube algorithm.

The Rubik's Cube algorithm was applied in sensitive information transmission schemes to encrypt information traffic on insecure physical channels [44]. There is also a cloud-based data protection mechanism based on the fingerprint algorithm, which encrypts a file by controlling the Rubik's Cube rotation, which depends on the feature value of a user's fingerprint [45].

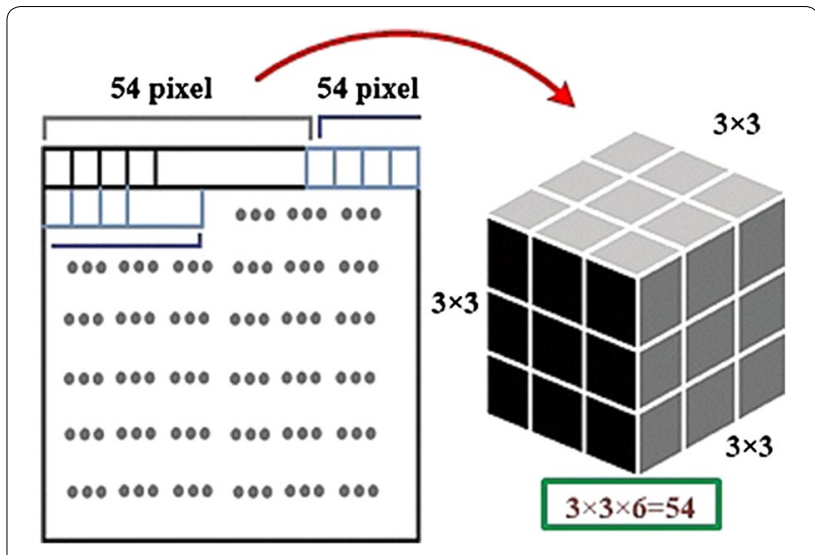

Figure 19 Mapping of Rubik's Cube and image

The rotation and permutation characteristics of the Rubik's Cube structure increase the anti-attack capability of a password. Thus, Rubik's Cube is very useful in the field of encryption, and became an ideal experimental prop to verify whether the robot operator can meet the initial standard.

In Ref. [46], the recovery of Rubik's Cube was used as a 3D operation task for a robot with different sensors (such as vision, force, torque, and tactile sensors). The controller performance of the robotic control system can be tested by the operation of restoring the Rubik's Cube [47], as shown in Figure 21, including movement, acceleration, delay, and orientation of the robot [48].

There is a method to solve the Rubik's Cube with a robot based on a noninvasive brain-computer interface without using the hands and only thinking about the Rubik's Cube rotation. This method improves the speed of solving and also helps disabled persons who cannot solve the Rubik's Cube with their hands [49].

Nowadays, with the intelligent development of science and technology, a robot can restore Rubik's Cube successfully, which means that intellectual technology is becoming more and more mature. The design of an intelligent robot that can automatically restore the Rubik's Cube from an arbitrary disruption involves digital image processing, computer vision, pattern recognition technology, artificial intelligence, organization, and other disciplines of technology. In the process of restoring the Rubik's Cube, the performance of robot can be detected, and the lack of a mechanism can be reflected as well. An appropriate way can be put forward to make up for deficiencies of the robot in time, and will promote the development of robot for higher intelligence.

Some concepts related to mechanical design have emerged, and some products that use the characteristics of the Rubik's Cube structure were invented as well. 

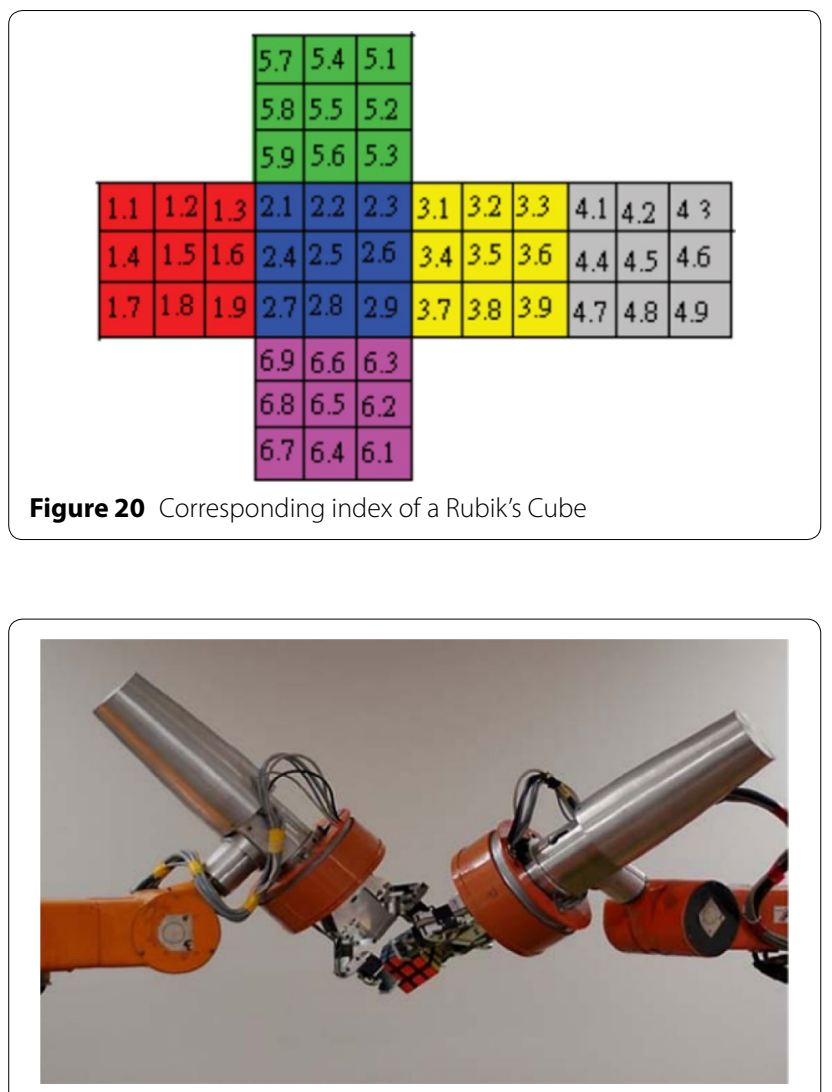

Figure 21 Two-handed system manipulating Rubik's Cube

Examples include a new password lock composed of a Rubik's Cube structure with a chin [50]. In Ref. [51], a metamorphic mechanism was designed, which used the cube as a platform for the operation of a machine and could be used in a multistructure. In addition, there are some ideas on the application of the cube in aerospace, such as a combination of the cube structure and a satellite, using the cube satellite to drive the cube unit location and orientation reconstruction in order to complete a variety of tasks [52]. Zhao proposed an application design of deformable magic-square-type deep-space exploration aircraft, applying the magic structural features of modularity and rotatability to a spacecraft, so that each box of the cube has a clear division of lab, and then through cooperation to complete the deep space exploration task [53]. Inspired by the ubiquitous Rubik's Cube toy, an equipment-free method was proposed for fabricating paper analytical devices [54].

\section{Application of Rubik's Cube Structure and Thinking in Mechanism}

Rubik's Cube, "Huarongdao" as invented by Chinese, and "Independent diamond" invented by the French are known as three major intellectual toys of the world
[55]. Although the Rubik's Cube was invented just over 40 years ago, it has been popular with people around the world, and an increasing number of cube enthusiasts and scholars have become hooked on Rubik's Cube's charm. The main reason is that Rubik's Cube contains the esoteric mathematical principles and an all-encompassing transformation, which attracts curious people to explore the mysteries of Rubik's Cube.

These researchers studied the description of rotation of the Rubik's Cube, explored recovery algorithms and the mathematical principles of the Rubik's Cube, and used the Rubik's Cube as a model to study the scientific problems in multidisciplines. As seen from the above studies, with the research and application of Rubik's Cube, it is not only an intellectual toy. It has changed from a research object to an object of significance in multidisciplinary research. These studies and applications are mainly based on the abstract characteristics of the Rubik's Cube structure. The research to explore the internal mechanism law of Rubik's Cube has just begun. The mechanism principle of Rubik's Cube structure has yet to have an in-depth study.

\subsection{Toy Structure and Mechanism}

Some new theories of the existing mechanism were inspired by handicrafts or exquisite toys, and were innovated through relevant research.

Wei [56] analyzed the degree of freedom of the chromospheric mechanism with a loop screw equation. In Ref. [57], the degree of freedom of the magic ball mechanism was analyzed based on the theory of structural decomposition and the system of equivalent spiral. The theory of a multiloop coupling mechanism was proposed by studying toys with coupling characteristics. Figure 22 shows the chromospheric mechanism and the magic ball mechanism.

Ding [58-60] used the snake cube to study the mathematical expression of mechanism constituent elements, the equivalence treatment of mechanism motion, the description of mechanism motion interference and isomorphism identification, and the sequence definition of a reconfigurable mechanism that can achieve a target

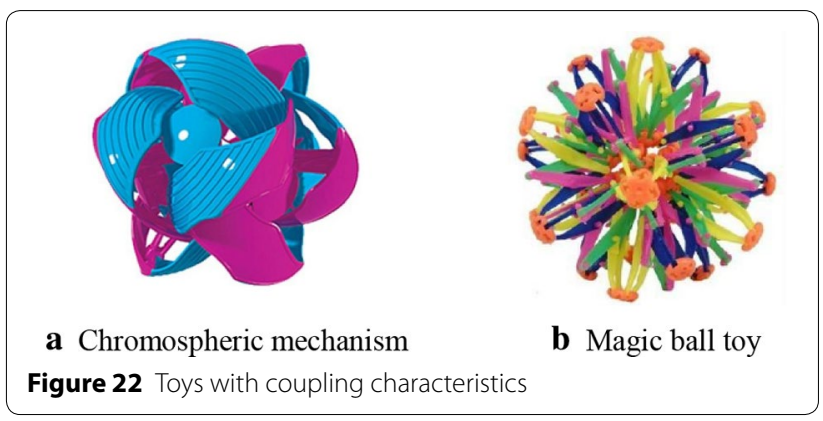


configuration. In Ref. [61], the position solution of the target configuration was calculated. Research on the snake cube enriched the modular reconfigurable mechanism theory, as shown in Figure 23.

Kuo [62] analyzed the reconfigurable cube mechanism, as shown in Figure 24. It is a puzzle linkage made by eight connected subcubes and is equivalent to a single-loop $8 \mathrm{R}$ spatial linkage with eight different topological configurations and changeable mobility.

Hsiao analyzed the structures of open-keyhole puzzle locks, which were widely used with plentiful types in ancient China. Open-keyhole puzzle locks, as shown in Figure 25, are reconfigurable mechanisms that have different topological structures in operation [63].

Dai et al. [64, 65] proposed a theory of metamorphic mechanism from research on origami handicrafts. This has aroused the interest of academia and has become one of the latest topics in the mechanics field.

A metamorphic mechanism has characteristics including a variable degree of freedom and topology, and can be applied to multiple operating conditions. Such mechanisms can be suitable for unstructured environments, variable conditions, and multitasking [66]. Lee and Herve proposed a discontinuous freedom link mechanism that extends from the principle of the Rubik's Cube, and has the property of a variable number of bars and degrees of freedom [67].

It was found that some properties of the Rubik's Cube mechanism are similar to metamorphic mechanisms through the analysis of the Rubik's Cube, and some properties are similar to multiloop coupling mechanisms. Examples include the characteristic of the coupling between the inner loop and the kinematic chain of the multiloop coupling mechanism, and the movement bifurcation characteristic of the change of the degree of freedom and the change of the motion after the special position. It can be seen that the Rubik's Cube has a variety of mechanism characteristics and is a combination of many kinds of mechanism characteristics, but this is not a simple superposition of the above mechanisms.

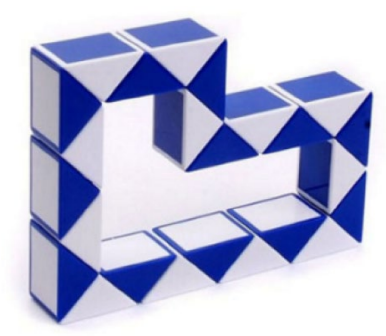

Figure $\mathbf{2 3}$ Structure of snake cube

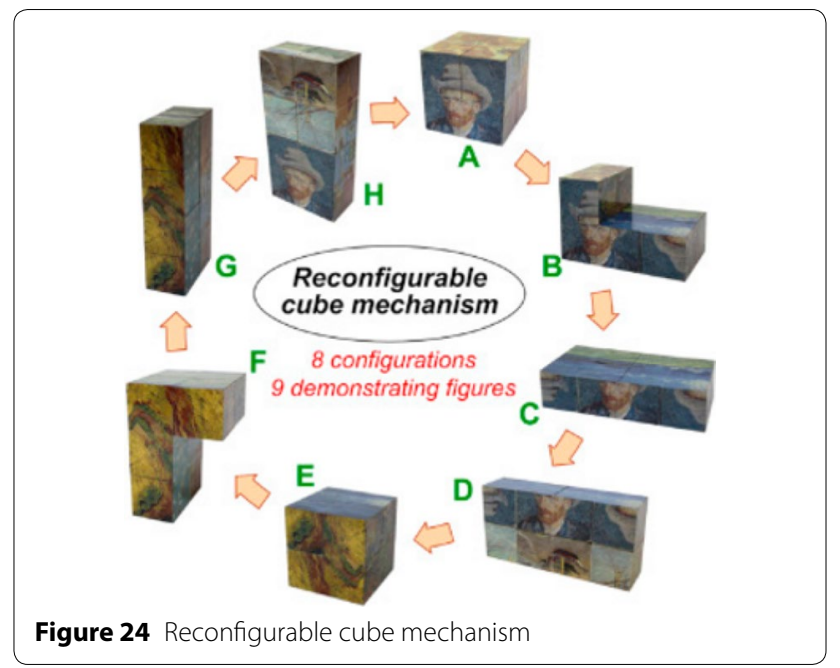

The structure of the Rubik's Cube is smart, simple, compact, and ever changing. To some extent, it goes beyond the traditional mechanisms of the concept of topology and conventional institutional design methods. The Rubik's Cube has been applied in daily life, and scholars have conducted studies of the cube problem in the field of mechanism. Only deep study and analysis of the cube structure can achieve the Rubik's Cube mechanism topology theory systematically.

\subsection{Application Outlook of Rubik's Cube in Mechanism}

With the continuous improvement in the demand of mechanical products, classical mechanisms are moving forward to modern complex mechanisms, which changed from fixed topology to variable topology and from weak coupling to strong coupling. Variable topology and strong coupling of the cube body gradually attracted attention. Compared with a parallel mechanism and simple multiloop coupled mechanism, the structure of Rubik's Cube has a higher complex degree of freedom, the rack of Rubik's Cube structure is connected to a number of end-effectors, and the connection between rod and rod is noncontinuous. However, Rubik's Cube has great

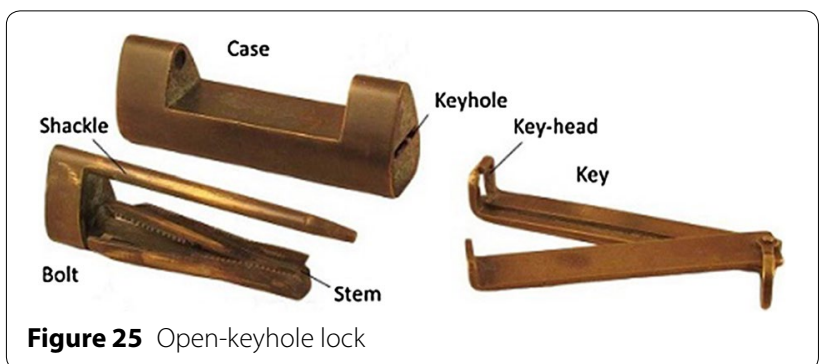


application potential in the industrial field for its features, including high space utilization, a large number of combinations, sophisticated sports form, and multimodule structure.

The application of Rubik's Cube in the machinery industry has the following three ideas:

(1) The multi-end effectors of Rubik's Cube and the ability of permutation and combination can be applied to mechanical design. The Rubik's Cube mechanism can be used as the main structure of multifunctional mechanical products. The use of the rotation of the cube mechanism can achieve the desired position or form of movement, so that different ends of the actuator can complete a task in order in a certain position, or make different ends of the actuator work together to complete a job with orderly cooperation.

(2) According to the kinetic pair characteristics of the Rubik's Cube structure that are different from the common structure, the cube kinetic pairs can be integrated into the current industrial mechanism. If such a clever design application can replace the robot spherical joint, the problem of a small rotation angle of the spherical joint being limited by the mechanical structure will be solved, thus expanding the robot's work space, especially that of the parallel mechanism. If this design can be applied to a machine tool holder or the manipulator of a mechanical arm, the flexibility of clamping and operation will be greatly improved.

(3) The Rubik's Cube structure has high space utilization. If this characteristic can be applied to other mechanical products, it will promote the miniaturization process of mechanical products.

In addition, with the development of the world's aerospace industry, there will be more and more deep-space exploration projects. Some features of the Rubik's Cube structure including modular, rotating, multifunctional, multipurpose, and recyclable functions can be applied to the design of deep-space exploration spacecraft or vehicles.

According to the above application ideas, the application of the cube structure also requires basic theoretical research. The movement of the product to achieve a certain direction involves the research of degrees of freedom in order to achieve the required form of movement and specific state related to the structural mathematical expression and the metamorphic properties. There are some problems with the Rubik's Cube mechanism, including a certain degree of flexibility, a large number of surface contacts, a number of movement directions of special locations, and many more. Subsequent research on specific static, friction, control, and other topics will be involved.

With further exploration, potential applications of the Rubik's Cube require the study of mechanism problems of the cube structure. This can lay a theoretical foundation for applications of the cube mechanism and promote special cube mechanisms from educational toys to machinery (such as robots, aerospace, etc.).

\section{Conclusions}

There are many types of Rubik's Cube. Some achievements in theoretical research and application have been made. This paper systematically introduced the origin and development of Rubik's Cube, analyzed the characteristics of structure and performance of Rubik's Cube, and examined the research status of Rubik's Cube, including science metaphors, restoration algorithms, and characteristic applications.

The external characteristics of Rubik's Cube have been studied and applied in multidisciplinary fields, so the principles of the internal structure of Rubik's Cube should be explored at the same time.

This paper described the relationship between Rubik's Cube and the mechanism. The research into Rubik's Cube in the field of mechanism is still in the initial stages. The new problems of the Rubik's Cube mechanism should be studied, and a systematic theory of the Rubik's Cube mechanism should be formed. Some achievements of the research have a guiding significance in the development of the Rubik's Cube mechanism in mechanical engineering applications.

It is of great significance to study the cube mechanism and to promote the development of the cube structure. The topology theory of the cube mechanism has yet to be further studied. It can be believed that Rubik's Cube will have extensive prospects for applications in the machinery industry based on its research status, and a spillover effect in some scientific research including mathematics, physics, computers, and biology.

\section{Authors' Contributions}

$\mathrm{D}-\mathrm{XZ}$ and $\mathrm{ZH}$ were in charge of the whole trial; $\mathrm{ML}$ and J-JW wrote the manuscript; Y-LH and W-JL assisted with sampling and laboratory analyses. All authors read and approved the final manuscript.

\footnotetext{
Authors' Information

Da-Xing Zeng, born in 1978, is currently a professor at Yanshan University, China. He received his PhD degree in mechatronic engineering from Yanshan University, China, in 2008. His research interests include theory of mechanism and machine, parallel mechanism, type synthesis and image processing. Ming Li, born in 1992, is currently a master candidate at Yanshan University, China. His research interests include Rubik's cube analysis, parallel mechanism and type synthesis.

Juan-Juan Wang, born in 1991, is currently a master candidate at Yanshan University, China. Her research interest is parallel mechanism.
} 
Yu-Lei Hou, born in 1980, is currently a professor at Yanshan University, China. His research interests include parallel mechanism, multi-axis force sensor, bionics of human robot, etc.

Wen-Juan Lu, born in 1983, is currently an associate professor at Yanshan University, China. Her research interests include parallel mechanism, type synthesis, etc.

Zhen Huang, born in 1936, is currently a professor and a PhD candidate supervisor at School of Mechanical Engineering, Yanshan University, China. His main research interests include parallel robot, type synthesis and topology.

\section{Competing interests}

The authors declare that they have no competing interests.

\section{Funding}

Supported by National Natural Science Foundation of China (Grant No. 51775473), Hebei Provincial Natural Science Foundation of China (Grant No. E2018203140), Hebei Provincial System Construction Project of Guide Program for Technology Innovation (Grant No. 18241905D)

\section{Publisher's Note}

Springer Nature remains neutral with regard to jurisdictional claims in published maps and institutional affiliations.

\section{Received: 24 August 2017 Accepted: 8 August 2018}

Published online: 27 August 2018

\section{References}

[1] D Gebhardt, G Hellings, W H Huang, et al. The cube. New York: Black Dog \& Leventhal Publishers, 2009.

2] T de Castella. The people who are still addicted to The Rubik's Cube. BBC News Magazine. BBC, 2014 [2018-05-30]. http://www.bbc.com/news/ magazine-27186297.

[3] Daily Mail Reporter. 'Driven Mad'Rubik's Nut Weeps on Solving Cube... after 26 Years of Trying, 2009. http://www.dailymail.co.uk/news/article-11123 96/Driven-mad-Rubiks-nut-weeps-solving-cube--26-years-trying.html.

[4] V D Stephen. Inventing the 20th Century: 100 Inventions That Shaped the World. New York: New York University Press, 2002.

[5] T Jerome. Rubik's Cube 25 Years on: Crazy Toys, Crazy Times. London: The Independent, 2007. https://www.independent.co.uk/news/science/rubik s-cube-25-years-on-crazy-toys-crazy-times-5334529.html .

[6] Carlisle, P Rodney. Encyclopedia of Play in Today's Society. Thousand Oaks: SAGE Publications Inc, 2009

[7] Europa. Interview with Ernö Rubik, 2016. http://www.create2009.europ a.eu/ambassadors/profiles/erno_rubik.html.

[8] J Ori. How Do You Beat the Rubik's Cube?, 2017. https://ourpastimes.com/ do-beat-rubiks-cube-6508960.html.

[9] J Lee. Beginner Solution to the Rubik's Cube., 2008. http://peter.stillhq.com/ jasmine/rubikscubesolution.html.

[10] Rubik's Cube Solver, 2016. https://rubiks-cube-solver.com.

[11] Rubik's Cube: A Question, Waiting to be Answered, 2014. https://www.youtu be.com/watch? $v=W 1 K 2 j d j L h b o$.

[12] SC Li. The science and culture in Rubik's Cube. Beijing: Higher Education Press, 2015. (in Chinese)

[13] J J Liu. Eastern origination of combinatorics. Journal of Northwest University (Natural Science Edition), 2001, 31(5): 457-460. (in Chinese)

[14] C Bandelow. Inside Rubik'S Cube and Beyond. Boston: Publisher Birkhauser Boston Inc., 1980.

[15] A Liszewski. You Probably Won't Live Long Enough to Solve the World's Largest 22x22 Rubik's Cube, 2016. https://gizmodo.com/you-probably-wontlive-long-enough-to-solve-the-worlds-1753163636.

[16] Greg's Puzzles. World Record 33×33×33 Rubik's Cube, 2017. https://www. youtube.com/watch? $v=N q J C 3 Y \times f c y M$

[17] Sheng Shou Puzzles. China Magic Cube. 2014. http://www.china-magic -cube.com/category/2.html.

[18] E Rubik, TVarga, G Kéri, et al. Rubik's Cubic compendium. New York: Oxford University Press. 1987
[19] R Hod. Finding the total number of legal permutations of the Rubik's Cubic. Trondheim: Trondheim Katedralskole, 2010.

[20] J M Wang, J P Fang, N Zhang. The application of combination creation in the shape design of the Rubik's Cubic. Art Panorama. 2013(6): 105-105. (in Chinese)

[21] S David. Notes on Rubik's Magic Cube. Enslow Publishers, 1981.

[22] A H Frey, S David. Handbook of cubic math. Enslow Publishers, 1982.

[23] D Joyner. Adventures in group theory: Rubik's Cube, Merlin's machine and other mathematical toys. John Hopkins University Press, 2002.

[24] J Chen. Group theory and the Rubik's Cube. ResearchGate, 2006.

[25] D Hasan, W Sumpun. Flower-like Ag/AgCl microcrystals: Synthesis and photocatalytic activity. Materials Chemistry and Physics, 2015, 159: 71-82.

[26] G H Jiang, X H Wang, Y Zhou et al. Hollow $\mathrm{TiO}_{2}$ nanocages with Rubik-like structure for high-performance photocatalysts. Materials Letters, 2012, 89: 59-62.

[27] J D Aten. The Rubik's Cube: A therapeutic metaphor. Journal of Psychology \& Christianity, 2004, 23(3): 258

[28] J He, KY Hu. Guide participation let the thinking sublimation in mathematical activities- the teaching guidance of "Playing Rubik's Cube" in primary school mathematics activity courses. Education Science Forum. 2011(2): 42-45. (in Chinese)

[29] T Davis. Teaching mathematics with Rubik's Cube. The Two-Year College Mathematics Journal, 1982, 13(3): 178-185. (in Chinese)

[30] Y Zheng. The theory of Rubik Cube and its applications. Hangzhou: Zhejiang University, 2009. (in Chinese)

[31] E Berlekamp, J H Conway, R K Guy. Winning ways for your mathematical plays. London: Academic Press. 1982.

[32] B Patrick. You can do the cube. London: Puffin Books. 1981.

[33] J Palmer. Cracking the last mystery of the Rubik's cube. New Scientist, 2008, 199(2668): 40-43.

[34] R E Korf. Learning to solve problems by searching for macro-operators. Pitman Advanced Pub. Program, 1985

[35] T Rokicki, H Kociemba, M Davidson, et al. The diameter of the Rubik's Cube Group is twenty. SIAM Journal on Discrete Mathematics, 2013, 27(2): 1082-1105.

[36] H B Ma, L H Liu. Design of Rubik's Cube permutation algorithm based on chaos. Computer Engineering and Applications, 2006, 42(12): 138-140. (in Chinese)

[37] L Zhang, S M Ji, Y Xie, et al. Principle of image encrypting algorithm based on magic cube transformation. Lecture Notes in Computer Science, 2005, 3802: 977-982.

[38] J Shen, X Jin, C Zhou. A color image encryption algorithm based on magic cube transformation and modular arithmetic operation. Lecture Notes in Computer Science, 2005, 3768: 270-280.

[39] G J Bao, J I Shi-Ming, J B Shen. Magic cube transformation and its application in digital image encryption. Computer Applications, 2002, 26(1): 7-22. (in Chinese)

[40] L L Zhao, Z L Fang, Z C Gu. A novel algorithm of digital image scrambling and encryption based on magic cube transformation. Journal of Optoelec tronics Laser, 2008, 19(1): 131-134. (in Chinese)

[41] K A Abitha, P K Bharathan. Secure communication based on Rubik's Cube algorithm and chaotic baker map. Procedia Technology, 2016, 24: 782-789.

[42] E Yen, L H Lin. Rubik's Cube watermark technology for grayscale images. Expert Systems with Applications, 2010, 37(6): 4033-4039.

[43] C L Tsai, C J Chen, W L Hsu. Multi-morphological image data hiding based on the application of Rubik's Cubic algorithm. 2012 IEEE International Carnahan Conference on Security Technology, 2012: 135-139.

[44] J L Li, G L Huo, B Liu. Sensitive information transmission scheme based on magic cube algorithm in automated trust negotiation. Journal of Computer Applications, 2011, 31(4): 984-988. (in Chinese)

[45] HWu, J L Fan, J H Liu. Cloud storage data protection mechanism based on a fingerprint cube algorithm. Telecommunications Science, 2014, 30(11): 110-115. (in Chinese)

[46] W Szynkiewicz. Skill-based bimanual manipulation planning. Journal of Telecommunications \& Information Technology, 2012, 2012(4): 54-62.

[47] C Zieliński, M Staniak, W Czajewski, et al. Rubik's Cube as a benchmark validating MRROC++ as an implementation tool for service robot control systems. Industrial Robot, 2007, 34(5): 368-375.

[48] S Kopácsi. Interactive visualisation in 3D. Journal of Physiology, 2012, 384(1): 671-690 
[49] M R Daliri, H Samadi. Solve the Rubik's Cube with robot based on noninvasive brain computer interface. 2014 Iranian Conference on Intelligent Systems (ICIS), 2014

[50] XX Miao. Rubik's Cube for Packaging: CN, 201510159029.9. 2015-04-07. http://www.patexplorer.com/patent/view.html?patid=CN20151015 9029.9\&sc $=\& q=\% E 7 \% 94 \% A 8 \% E 4 \% B A \% 8 E \% E 5 \% 8 C \% 85 \% E 8 \% A 3 \% 85 \%$ E7\%89\%A9\%E7\%9A\%84\%E9\%AD\%94\%E6\%96\%B9\&fq=\&sort=\&sortF ield $=\&$ page $=1 \&$ rows $=10 \# 1 / C N 201510159029.9 /$ detail/abst. (in Chinese)

[51] R G Wang, Y F Liao, C Zhang. An obstacle avoidance machine crab with metamorphic mechanism: CN: 201510119249.9. 2015-06-17. http://www. patexplorer.com/patent/view.html?patid $=\mathrm{CN} 201510119249.9 \& \mathrm{sc}=\& q=$ \%E4\%B8\%80\%E7\%A7\%8D\%E5\%8F\%98\%E8\%83\%9E\%E6\%9C\%BA\%E6\%9 E\%84\%E5\%BC\%8F\%E5\%8F\%AF\%E8\%B6\%8A\%E9\%9A\%9C\%E6\%9C\%BA\% E5\%99\%A8\%E8\%9F\%B9\&fq=\&sort=\&sortField=\&page=1\&rows=10\#1/ CN201510119249.9/deta. (in Chinese)

[52] B J Cui. Cube satellite and its design method: CN, 2013107194458.8 2014-04-16. http://www.patexplorer.com/patent/view.html?patid $=$ CN201310719458.8\&. (in Chinese)

[53] B H Zhao, SY Gao, J N Zhang, et al. Rubik's Cube type deep space exploration aircraft. Technology Innovation and Application, 2014(24): 61-63. (in Chinese)

[54] H Fu. Using the Rubik's Cube to directly produce paper analytical devices for quantitative point-of-care aptamer-based assays. Biosensors and Bioelectronics, 2017, 96: 194-200.

[55] Y Chen, L Jiang. Analysis of the application of Rubik's Cube in teaching. China Electric Power Education, 2012(8): 88-89. (in Chinese)

[56] G Wei, X Ding, J S Dai. Mobility and geometric analysis of the Hoberman Switch-Pitch Ball and its variant. Journal of Mechanisms \& Robotics, 2010, 2(3): 191-220.

[57] J S Dai, D Li, Q Zhang, et al. Mobility analysis of a complex structured ball based on mechanism decomposition and equivalent screw system analysis. Mechanism and Machine Theory, 2004, 39(4): 445-458.
[58] X L Ding, S N Lü, Y Yang. Configuration transformation theory from a chain-type modular reconfigurable mechanism-Rubik's Snake. The 13th World Congress in Mechanism and Machine Science, México, Guanajuato: IFToMM, 2011: A12_380.

[59] X L Ding, S N Lu. Reconfiguration theory of modular reconfigurable mechanism based on analysis of snake cube. Journal of Mechanical Engineering, 2012, 48(11): 126-135. (in Chinese)

[60] X Ding, S Lu. Fundamental reconfiguration theory of chain-type modular reconfigurable mechanisms. Mechanism and Machine Theory, 2013, 70(Supplement C): 487-507. (in Chinese)

[61] CWang, J J Xu. An algorithm for finding the three-dimension paths of the S-shaped intellectual magic square. Computer Knowledge and Technology, 2008, 1(4): 686-690.

[62] C H Kuo, J W Su. Configuration analysis of A class of reconfigurable cube mechanisms: Mobility and configuration isomorphism. Mechanism and Machine Theory, 2017, 107: 369-383.

[63] K H Hsiao. On the structural analysis of open-keyhole puzzle locks in Ancient China. Mechanism and Machine Theory, 2017, 118: 168-179.

[64] J S Dai, J R Jones. Mobility in metamorphic mechanisms of foldable/ erectable kinds. Journal of Mechanical Design, 1999, 121(3): 375-382.

[65] D L Li, Z H Zhang, J S Dai. Overview and prospects of metamorphic mechanism. Journal of Mechanical Engineering, 2010, 46(13): 14-21. (in Chinese)

[66] Department of Engineering and Materials Science, National Natural Science Foundation of China. The Research Report of Disciplines Development Strategy (2011-2020): Mechanical Engineering Discipline. Beijing: Science Press Ltd, 2010. (in Chinese)

[67] C C Lee, J M Herve. Discontinuous mobility of four-link mechanisms with revolute, prismatic and cylindrical pairs through the group algebraic structure of the displacement set. The 8th International Conference on the Theory of Machines and Mechanisms, Czech Republic: Liberec Elsmere, 2001: 5-7.

\section{Submit your manuscript to a SpringerOpen ${ }^{\circ}$ journal and benefit from:}

- Convenient online submission

- Rigorous peer review

- Open access: articles freely available online

- High visibility within the field

- Retaining the copyright to your article

Submit your next manuscript at springeropen.com 